\title{
Hand Trauma: "gradient of pressure" technique to block the radial and medial nerves
}

Karl Otto Geier MD, MSc ${ }^{1}$ Department of Pain Clinic Hospital São Lucas Faculdade de Medicina da Pontificia Universidade Católica (PUCRS), RGS-Brazil; ${ }^{2}$ Department of Anesthesiology Hospital Municipal de Pronto Socorro de Porto Alegre, RGS-Brazil;Adress : Rua

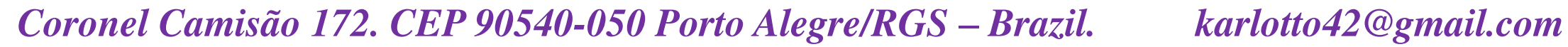

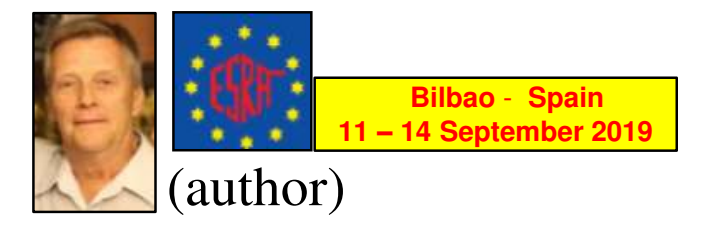

\section{BACKGROUND and AIMS}

In hand trauma the first indication lies on axillary brachial plexus anesthesia. However, many patients report discomfort by loss of identity of the limb. Distal hand block eliminated this discomfort with motor maintenance, useful in tendon sews $(1,2)$. The digital "gradient of pressure" (GOP) was used in four cases.

\section{RESULTS}

The medial nerve run between the pronator teres and flexor digitorum superficialis muscles and the radial nerve between the brachial and brachioradial muscles (Fig.2). Both were blocked in they fascial muscle spaces. Latency, $20 \mathrm{~min}$; analgesic, 9 hours; digital movements preserved.

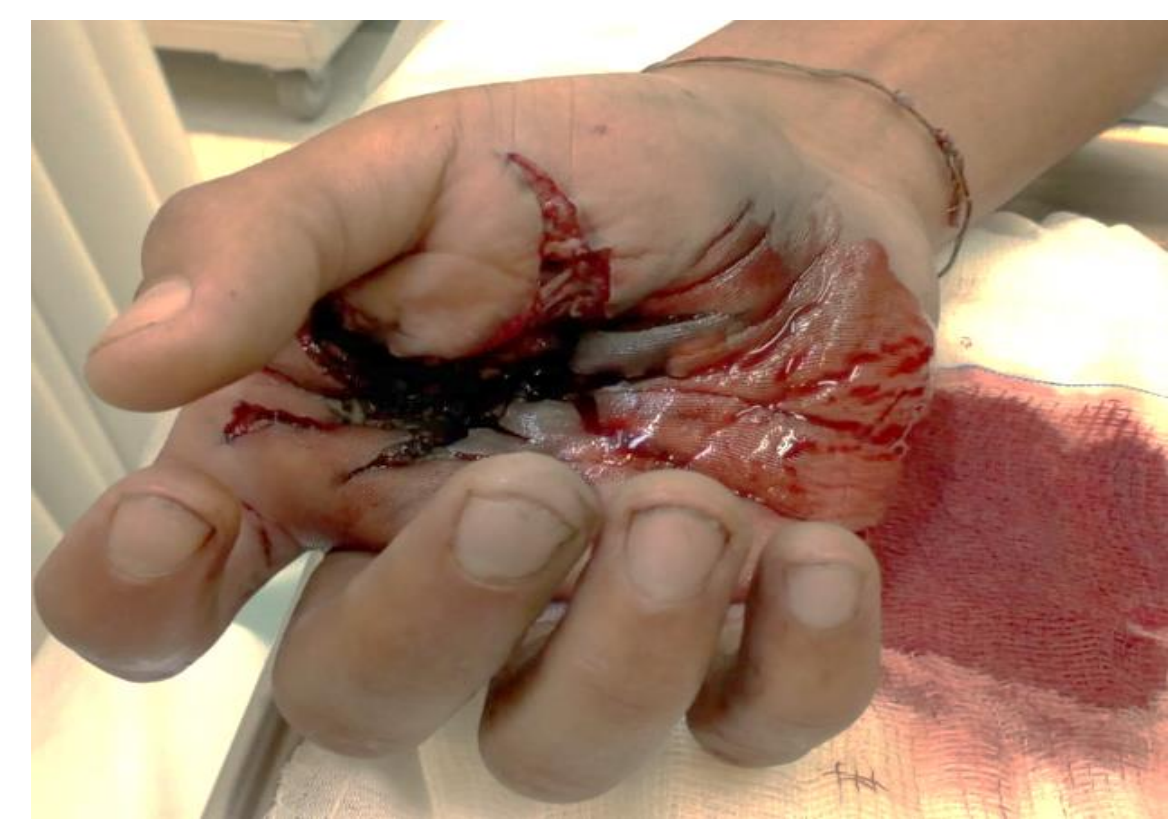

Figure 1

\section{METHODS}

Male patient, 28 y-old, ASA I, had his hand detonated by an explosion (Fig.1). At the hospital under scalene brachial block carried out by the anesthesiologist on duty, the entire limb resulted numb. During stay at the Infirmary multiple cleanings were required with IV morphine, triggering tolerance. A new debriment was necessary under general GA. In the RR, with pain NPS $=8$, patient refuted new numbed limb consenting to analgesia of the hand with single $6 \mathrm{ml}, 2 \%$ of lidocaine at each distal involved nerve (Fig.2). The GOP technique was used in both, median and radial nerves.

\section{CONCLUSION}

Both, GOP and LOR techniques are useful when PNS, US aren't available. They are based on anatomic references and digital feeling when the needle identifies sudden absence of resistance (epidural space) or decrease resistance (fascial spaces), respectively. Surgical tendon sewing under analgesia with preserved movements may be useful to plastic surgeries $(3,4)$.
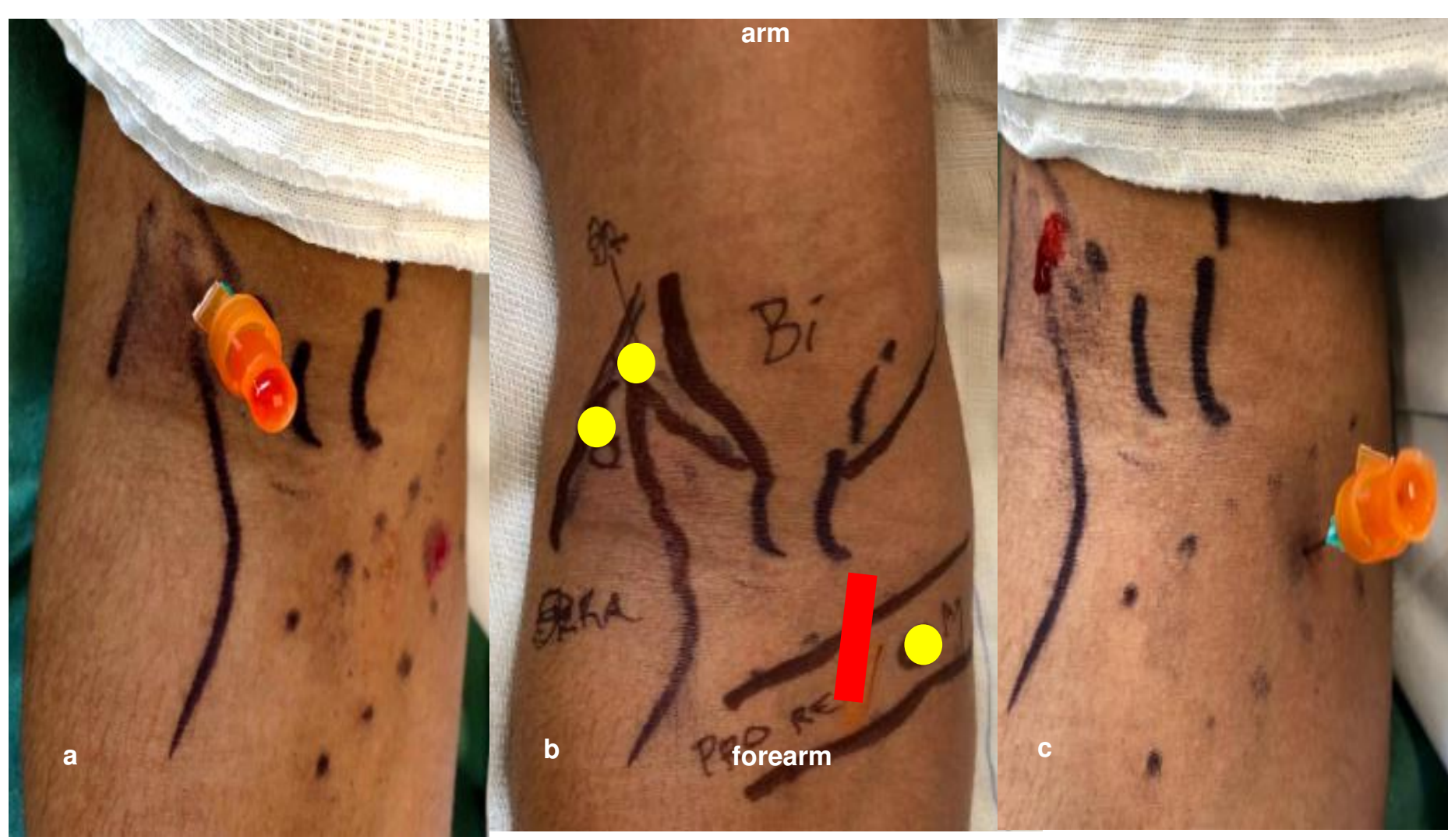

Figure 2.- a)- Radial nerve block needle trans brachial Muscle; b)- puncture landmarks; c)- Median nerve block through the pronator teres muscle. Note that in both there are reflux of LA. Puncture sites (Yellow circles); Ulnar artery (red line).

References 1.- Geier KO. Prolonged Regional Analgesia with Peripheral Catheters. Case Reports Rev Bras Anestesiol 2002;52:1:68-73 ; 2.- Singh S K. Just A Nee-dle and Some Local Anaesthetic to Relieve Acute Pain, The Loss of Resistance Blocks. Journal of Anaesthesia and Critical Care Case Reports July-Sep 2015:1(1): 4-5.; 3.- Tang JB. Wide-awake primary flexor tendon repair, tenolysis and tendon transfer. Clin Orhop Surg 2015; 7: 275-81; 4.- Mehlmann FM et al. Ultrasound-guided selective nerve blocks for trigger finger surgeries to maintain flexion/extension of fingers - Case series. Rev Bras Anestesiol, 2019 in Press (in English) 